\title{
Measurement of the magnetic moment of the negative muon bound in heavy atoms
}

\author{
T. N. Mamedov, * K. I. Gritsay, and A. V. Stoykov ${ }^{\dagger}$ \\ Joint Institute for Nuclear Research, 141980 Dubna, Moscow region, Russia \\ D. Herlach, R. Scheuermann, and U. Zimmermann \\ Paul Scherrer Institut, CH-5232 Villigen PSI, Switzerland
}

(Received 1 January 2007; revised manuscript received 28 February 2007; published 7 May 2007)

\begin{abstract}
The magnetic moment ( $\mathrm{g}$ factor) of the negative muon bound in the $1 s$ state of $\mathrm{Zn}, \mathrm{Ge}, \mathrm{Cd}$, and $\mathrm{Pb}$ atoms was measured. A considerable difference between the present experimental results and that of theoretical calculations and results from earlier measurements is observed.
\end{abstract}

DOI: $10.1103 /$ PhysRevA.75.054501

PACS number(s): 36.10.Dr, 31.30.Jv

As follows from theoretical calculations [1-3] and experimental investigations [4-8], the magnetic moment of the negative muon bound in the $1 s$ state of an atom differs substantially from that of the free muon. The principal correction to the magnetic moment of the negative muon arises from its relativistic motion in the Coulomb field of the nucleus. In the pointlike nucleus approximation the relativistic correction to the muon $g$ factor depends on the nuclear charge $Z$ as [1]

$$
a_{\mu}^{1 s}=\left(g_{\mu}^{\text {free }}-g_{\mu}^{1 s}\right) / g_{\mu}^{\text {free }}=\frac{2}{3}\left(1-\sqrt{1-\alpha^{2} Z^{2}}\right),
$$

where $g_{\mu}^{\text {free }}$ and $g_{\mu}^{1 s}$ are the $g$ factors of the free muon and the muon bound in the $1 s$ state of an atom and $\alpha$ is the fine structure constant.

The relativistic correction to the magnetic moment of the electron in the $1 s$ state of hydrogen and hydrogenlike ions of light atoms $\left(\mathrm{He}^{1+}, \mathrm{C}^{5+}, \mathrm{N}^{6+}\right.$, and $\left.\mathrm{O}^{7+}\right)$ was measured with high accuracy [9-12]. There is an excellent agreement between experimental results and theoretical calculations. The correction to the magnetic moment of the negative muon bound in an atom differs from that for the electron due to the finite size of the nucleus and the much smaller Bohr radius of the muon in the $1 s$ state $(\simeq 1 / 206$ of the Bohr radius of the $1 s$ electron) [3]. This difference is more substantial for medium and heavy atoms, as in atoms with $Z>30$ the muon Bohr radius is comparable with the size of the nucleus.

The experimental data on $a_{\mu}^{1 s}$ for atoms with $Z \lessgtr 20$ are consistent and agree with the theoretical calculations. For higher $Z$ atoms, such as $\mathrm{Zn}$ and $\mathrm{Cd}$, the data obtained in Refs. $[6,7]$ disagreed with the predictions of the theory [3] by more than five standard deviations but in a recently published paper [8] this result was not confirmed. This moved us to carry out additional precision measurements for medium and high $Z$ atoms.

The measurements were performed at room temperature $(T \simeq 300 \mathrm{~K})$ on the same $\mathrm{Zn}$ and $\mathrm{Cd}$ samples studied earlier [7], as well as on three new high purity samples: Zn (abun-

\footnotetext{
*Electronic address: tmamedov@nu.jinr.ru

${ }^{\dagger}$ Also at Paul Scherrer Institut, CH-5232 Villigen PSI, Switzerland.
}

dance of the main element is 0.9999$)$, Ge (overall concentration of impurities less than $1 \mathrm{ppm}$ ), and $\mathrm{Pb}$ (abundance of the main element is 0.99999). As a reference, a graphite sample was also measured. The high purity samples were used to minimize the possible contribution of the Knight shift to the measured muon spin precession frequency. From this point of view, the measurement in $n$-type single crystal germanium (concentration of As impurity $1.5 \times 10^{15} \mathrm{~cm}^{-3}$; resistivity $0.6 \mathrm{Ohm} \mathrm{cm}$ at $T=300 \mathrm{~K}$ ) is most important. Germanium (as well as silicon) with covalent chemical bonds is a diamagnetic material. At room temperature the concentration of free charge carriers is at least by 6 orders of magnitude lower than in a metal. Therefore, in germanium the value of the Knight shift should be reduced to zero. This conclusion is in good agreement, for example, with the results of NMR measurements in boron doped silicon [13]. It is necessary to note that experimental data on the Knight shift in $\mathrm{Zn}, \mathrm{Cd}$, and $\mathrm{Pb}$ with impurity concentrations $\$ 2 \%$ are absent (see, for example Ref. [14]). Possible influence of the Knight shift on the results of measurements of the negative muon $g$ factor has been discussed in detail in Refs. [6,7].

The $g$ factor of the muon was determined by the measurement of the muon spin precession frequency in an external magnetic field. The frequency of the muon spin precession was extracted from the time distribution of electrons from the decay of muons stopped in the sample (the $\mu$ SR spectrum) measured with a time-to-digital converter (TDC). We used two types of TDCs: an ORTEC 9308 pTA picosecond time analyzer, and a CAEN V1190B (for the measurements with $\mathrm{Zn}$ and $\mathrm{Pb}$ samples). For the 9308 pTA we carried out an "offline" calibration using a Rohde \& Schwarz SML 01 precision quartz clock of a frequency of $100 \mathrm{MHz}$. The measured nonlinearity of the TDC was within $10^{-4}$, which matches to its specification.

The measurements were performed at the GPD spectrometer at the $\mu \mathrm{E} 1$ muon beamline of the Swiss Muon Source $(\mathrm{S} \mu \mathrm{S})$. The external magnetic field of $2.5 \mathrm{kOe}$ was created by Helmholtz coils. The fraction of muons stopped in the samples was at least $96 \%$, which is important for decreasing the background components in the experimental spectra.

The polarization of the $\mu \mathrm{E} 1$ muon beam is approximately $80 \%$ (at $60 \mathrm{MeV} / \mathrm{c}$ ), this is about a factor of 2 higher compared to the polarization of the muon beam in Ref. [8]. Note that the statistical error in the determination of the muon spin 

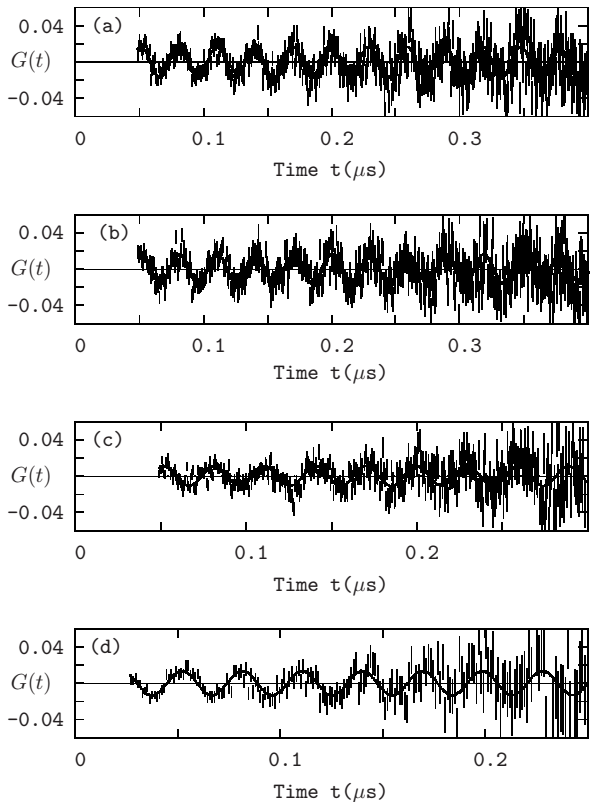

FIG. 1. Muon spin precession signals in $\mathrm{Zn} \mathrm{(a),} \mathrm{Ge} \mathrm{(b),} \mathrm{Cd} \mathrm{(c),}$ and $\mathrm{Pb}(\mathrm{d})$ measured in a magnetic field of $2.5 \mathrm{kOe}$ (the bin width is $1.25 \mathrm{~ns}$ for $\mathrm{Zn}, \mathrm{Ge}$, and $\mathrm{Cd}$ samples and $1.1718 \mathrm{~ns}$ for $\mathrm{Pb}$ ). The data are corrected for the muon lifetime in the $1 s$ state of the atom.

precession frequency from the experimental data is inversely proportional to the value of the muon polarization $P$ and the magnetic field $\Delta \omega / \omega \sim(P H \sqrt{N})^{-1}$, where $H$ is the magnetic field and $N$ is the number of collected events. The experiment [8] was performed at a magnetic field of $20 \mathrm{kOe}$.

For each sample the measurements were repeated two or three times. Four electron telescopes were used to detect muon decay events. Therefore, for each sample eight or more spectra of the time distribution of the decay electrons were obtained. For all the samples a clear muon spin precession signal in the transverse magnetic field was observed (see Fig. 1).

The experimental data were fitted in the time domain by the least-squares method using the function

$$
\begin{gathered}
N(t)=\sum_{i=1}^{2} N_{0 i}\left[1+G_{i}(t)\right] e^{-t / \tau_{i}}+b g, \\
G_{i}(t)=\frac{P_{i}}{3} \cos \left(\omega_{i} t+\varphi_{i}\right),
\end{gathered}
$$

where $N_{0 i}$ is proportional to the number of muons captured by atoms of type $i$ (apart from the sample, some fraction of muons stop in the scintillation counters); $\tau_{i}, P_{i}, \omega_{i}$ are, respectively, the muon lifetime, the muon spin polarization, and the spin precession frequency in the $1 s$ state of the atom; $\varphi_{i}$ is the initial phase of the muon spin precession; and $b g$ is the background.

In the $\mu \mathrm{E} 1$ muon beam there is an admixture of electrons with a time ("bunch") structure reflecting that of the proton beam of the accelerator. This allowed an "online" calibration of the setup under real operating conditions. The time structure of the proton beam is determined by the frequency of the

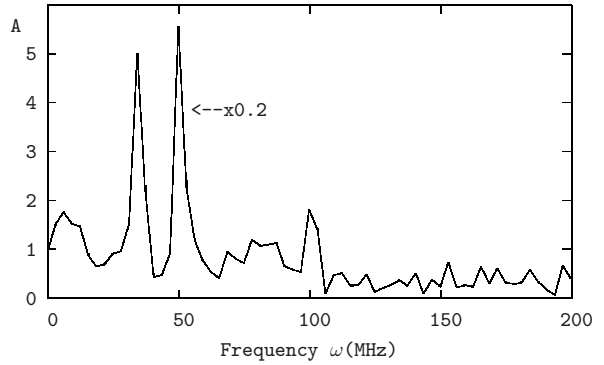

FIG. 2. Fourier transform of the transverse field experimental data for the Ge sample. The peak at 33.6 MHz corresponds to muon spin precession in the external magnetic field of $2.5 \mathrm{kOe}$. The peak at $50.63 \mathrm{MHz}$ is due to an admixture of electrons with "bunched" time distribution in the muon beam.

accelerating field set up by a high stability quartz generator $\left(f_{\mathrm{ac}}=50.6330 \mathrm{MHz}\right)$. The online calibration was done by fitting the bunch frequency in a set of time windows of $1.70 \tau$ ( $\tau$ is the muon lifetime for the given atom) in different parts of the $\mu$ SR spectra as $b g=b_{0}+\sum_{n} b_{n} \cos \left(2 n \pi f_{a c}+\varphi_{n}\right)$. Within the experimental accuracy of $3 \times 10^{-4}$ the bunch frequency was found to be equal to $f_{\text {ac }}$ in the whole TDC range. In Fig. 2 the result of a fast Fourier transformation of the $\mu \mathrm{SR}$ data for $\mathrm{Ge}$ is presented. The raw $\mu \mathrm{SR}$ data were corrected for the constant background and the muon lifetime in Ge $(\tau$ $=166.5 \mathrm{~ns}$ [15]). The bin width was $0.625 \mathrm{~ns}$ and the time window used for the fast Fourier transform was $(50-390)$ ns. Both the frequency corresponding to the muon spin precession in the external magnetic field of $2.5 \mathrm{kOe}$ and the bunch frequency at $50.63 \mathrm{MHz}$ are clearly seen.

Thus, the same spectra were used for determination of the muon spin precession frequency and for calibration of the TDC time scale. In total for the studied samples $48 \mu \mathrm{SR}$ spectra were fitted by the least square method to the function (2) with the $\chi_{i}^{2}$ values in the range $0.96-1.07$. The measured values of the muon spin precession frequency $\omega$ were used to calculate the correction $a_{\mu}^{1 s}$ to the muon magnetic moment as

$$
a_{\mu}^{1 s}=\left(g_{\mu}^{\text {free }}-g_{\mu}^{1 s}\right) / g_{\mu}^{\text {free }}=\left(\omega^{\text {free }}-\omega\right) / \omega^{\text {free }},
$$

where $\omega^{\text {free }}$ is the spin precession frequency of a free muon. It was determined from the precession frequency of the positive muon spin in copper in the same magnetic field, taking into account the Knight shift of $(60.0 \pm 2.5) \times 10^{-6}$ [16].

The measured values of the asymmetry coefficient $(P / 3)$ of the angular distribution of electrons from the decay of muons, $\omega$ and $a_{\mu}^{1 s}$ are given in Table I. As in Ref. [8], no corrections are introduced into the value of $a_{\mu}^{1 s}$. As is seen, for the carbon sample there is good agreement in the $a_{\mu}^{1 s}$ value between the results of different experiments and of theoretical calculations. At the same time our results for $\mathrm{Zn}$, $\mathrm{Cd}$, and $\mathrm{Pb}$ substantially differ (about eight standard deviations) from that of Ref. [8] (the present values of $a_{\mu}^{1 s}$ coincide within experimental errors with the ones obtained earlier in Refs. [6,7]). There is also a discrepancy between our experimental data and the theoretical calculations for $\mathrm{Zn}, \mathrm{Cd}$, and $\mathrm{Pb}$ atoms. The result of Ref. [8] for $\mathrm{Pb}$ also deviates from the theoretically predicted value. 
TABLE I. Measured frequencies and amplitudes of the spin precession and the $g$-factor shift (relative to the free muon) of the negative muon bound in various atoms. For the positive muon in copper the precession frequency is corrected for the value of the Knight shift [16].

\begin{tabular}{|c|c|c|c|c|c|c|}
\hline Sample & Frequency $(\mathrm{MHz})$ & $\mathrm{P} / 3(\%)$ & \multicolumn{4}{|c|}{$10^{4} \times\left(g_{\mu}^{\text {free }}-g_{\mu}^{1 \mathrm{~s}}\right) / g_{\mu}^{\text {free }}$} \\
\hline $\mathrm{Cu}\left(\mu^{+}\right)$ & $33.8772 \pm 0.0007$ & & & & & \\
\hline $\mathrm{C}$ & $33.8523 \pm 0.0013$ & $5.30 \pm 0.03$ & $7.3 \pm 0.4^{\mathrm{a}}$ & $7.5 \pm 0.2^{\mathrm{b}}$ & $7.18 \pm 0.23^{\mathrm{c}}$ & $8.2^{\mathrm{d}}$ \\
\hline $\mathrm{Zn}$ & $33.652 \pm 0.021$ & $1.73 \pm 0.05$ & $67 \pm 6^{\mathrm{a}}$ & $75 \pm 9^{b}$ & $115.0 \pm 2.6^{\mathrm{c}}$ & $129^{\mathrm{d}}$ \\
\hline $\mathrm{Zn}(0.9999)$ & $33.625 \pm 0.021$ & $1.68 \pm 0.05$ & $73 \pm 6^{\mathrm{a}}$ & & & \\
\hline $\mathrm{Ge}$ & $33.617 \pm 0.022$ & $1.69 \pm 0.04$ & $77 \pm 7^{\mathrm{a}}$ & & & \\
\hline $\mathrm{Cd}$ & $33.734 \pm 0.036$ & $1.10 \pm 0.04$ & $42 \pm 10^{\mathrm{a}}$ & $67 \pm 22^{b}$ & $215_{-21}^{+17 c}$ & $218^{\mathrm{d}}$ \\
\hline $\mathrm{Pb}(0.99999)$ & $33.625 \pm 0.084$ & $1.60 \pm 0.10$ & $75 \pm 25^{\mathrm{a}}$ & & $260_{-23}^{+22 \mathrm{c}}$ & $383^{\mathrm{d}}$ \\
\hline
\end{tabular}

${ }^{\mathrm{a} P r e s e n t}$ results.

${ }^{\mathrm{b}}$ Experimental data from Ref. [6].

${ }^{c}$ Experimental data from Ref. [8].

${ }^{\mathrm{d}}$ Theoretical calculation from Ref. [3].

As we already discussed in Refs. [6,7], the discrepancy between the results of the theoretical calculations and experiment could arise from the following: (a) the measurements are performed in a medium and the effect the medium is not taken into account in the calculations; (b) in the case of heavy atoms, when the muon Bohr radius is comparable with the radius of the nucleus, the theory fails to calculate the value of the correction to the negative muon $g$ factor.
The reason for the large discrepancy between our data and the results of Ref. [8] is not clear, but the performed offline and online calibrations imply that the systematic error in our measurements is negligible. It is worth mentioning that the present results for $\mathrm{Zn}, \mathrm{Cd}$, and $\mathrm{Pb}$ are based on approximately 12 times larger statistics than the results of Ref. [8].

We are grateful to the Directorate of the Paul Scherrer Institute for allowing us to carry out the experiment at PSI.
[1] G. Breit, Nature (London) 122, 649 (1928).

[2] H. Margenau, Phys. Rev. 57, 383 (1940).

[3] K. W. Ford, V. W. Hughes, and J. G. Wills, Phys. Rev. 129, 194 (1963).

[4] D. P. Hutchinson, J. Menes, G. Shapiro, and A. M. Patlach, Phys. Rev. 131, 1362 (1963).

[5] T. Yamazaki et al., Phys. Lett. 53B, 117 (1974).

[6] T. N. Mamedov et al., JETP Lett. 93, 941 (2001).

[7] T. N. Mamedov et al., Physica B 326, 15 (2003).

[8] J. H. Brewer, A. M. Froese, B. A. Fryer, and K. Ghandi, Phys. Rev. A 72, 022504 (2005).

[9] N. Hermanspahn, H. Häffner, H.-J. Kluge, W. Quint, S. Stahl,
J. Verdú, and G. Werth, Phys. Rev. Lett. 84, 427 (2000).

[10] H. Häffner et al., Phys. Rev. Lett. 85, 5308 (2000).

[11] J. L. Verdú et al., Phys. Rev. Lett. 92, 093002 (2004).

[12] S. Stahl et al., J. Phys. B 38, 297 (2005).

[13] R. K. Sundfors and D. F. Holcomb, Phys. Rev. 136, A810 (1964).

[14] Li Bai-Qin and Wang Ye-Ning, Phys. Rev. B 47, 16582 (1993).

[15] T. Suzuki, D. F. Measday, and J. P. Roalsvig, Phys. Rev. C 35, 2212 (1987).

[16] A. Schenck, Helv. Phys. Acta 54, 471 (1981). 